\title{
Exhaled volatile organic compounds and lung microbiome in COPD: a pilot randomised controlled trial
}

\author{
Divya Mohan $\mathbb{1}^{1}$, Holly R. Keir ${ }^{2}$, Hollian Richardson ${ }^{2}$, David Mayhew ${ }^{1}$, Joseph Boyer ${ }^{1}$, \\ Marc P. van der Schee ${ }^{3}$, Max D. Allsworth ${ }^{3}$, Bruce E. Miller $\mathbb{B}^{1}$, Ruth Tal-Singer $\mathbb{C}^{1}$ and James D. Chalmers ${ }^{2}$
}

${ }^{1}$ GSK R\&D, Collegeville, PA, USA. ${ }^{2}$ Ninewells Clinical Research Centre, University of Dundee, Dundee, UK. ${ }^{3}$ Owlstone Medical, Cambridge, UK.

Corresponding author: James D. Chalmers (j.chalmers@dundee.ac.uk)

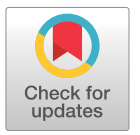

This version is distributed under the terms of the Creative Commons Attribution NonCommercial Licence 4.0. For commercial reproduction rights and permissions contact permissions@ersnet.org

Received: 11 April 2021

Accepted: 19 July 2021

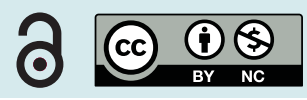

Shareable abstract (@ERSpublications)

VOC measurement in clinical trials to identify COPD subsets is feasible, but assessment of VOC technologies must include concurrent GC-MS validation. Further work to standardise collection of VOCs and measure a background or "housekeeper" VOC is required. https://bit.ly/3BNyKvS

Cite this article as: Mohan D, Keir HR, Richardson $\mathrm{H}$, et al. Exhaled volatile organic compounds and lung microbiome in COPD: a pilot randomised controlled trial. ERJ Open Res 2021; 7: 00253-2021 [DOI: 10.1183/23120541.00253-2021].

\section{Abstract}

Background Breath analysis is a burgeoning field, with interest in volatile organic compounds (VOCs) as a noninvasive diagnostic tool or an outcome measure, but no randomised controlled trials (RCTs) have yet evaluated this technology in a clinical trial longitudinally. In a pilot RCT, our exploratory objectives were feasibility of measuring VOCs via multiple techniques, assessing relationships between VOCs and Haemophilus colonisation and whether CXCR2 antagonism with danirixin altered lung microbiome composition in individuals with COPD.

Method 43 participants had VOCs and sputum biomarkers evaluated. VOCs and induced sputum were collected after $6 \mathrm{~h}$ of fasting at screening and at days 1, 7 and 14. VOCs were analysed via gas chromatography mass spectrometry (GC-MS), field asymmetric ion mobility spectrometry (FAIMS) and eNose. The primary outcome for these analyses was the relationship between VOCs and Haemophilus abundance determined by $16 \mathrm{~S}$ rRNA sequencing.

Results A joint-effects model demonstrated a modest relationship between four exhaled VOCs and Haemophilus relative abundance $\left(\mathrm{R}^{2}=0.55\right)$ measured only by GC-MS, but not as measured using gas chromtaography FAIMS or eNose. There was considerable variability in absolute quantities of individual VOCs longitudinally.

Conclusions VOC measurement in clinical trials to identify subsets of COPD is feasible, but assessment of new VOC technologies must include concurrent GC-MS validation. Further work to standardise collection of VOCs and measuring a background or "housekeeper" VOC is required to understand and normalise individual VOC quantities.

\section{Introduction}

Breath analysis via measurement of volatile organic compounds (VOCs) is a burgeoning and emerging field, with interest in the use of VOCs as a noninvasive tool for patient diagnosis and stratification or even as an outcome measure in clinical trials. One such example is fractional exhaled nitric oxide $\left(F_{\text {eNo }}\right)$, which has been successfully adopted into clinical and research practice [1]. Breath analyses have been shown to distinguish smokers from nonsmokers [2], and COPD subjects from non-COPD subjects [3], as well as relating to COPD severity [4] in cross-sectional sampling.

The lung microbiome may be an important disease modulator in COPD, where Haemophilus influenzae colonisation appears to increase neutrophilic inflammation, including formation of neutrophil extracellular traps (NETs) [5]. Although ordinarily a host defence mechanism [6], excessive NETs may cause host damage in airways disease [7], and may provide the mechanistic link between airway infection/ colonisation, airway inflammation and disease progression. Noninvasive diagnosis of airway infection and 
inflammation could enhance targeting of antibiotic or anti-inflammatory therapies such as CXCR2 antagonists, which have been shown to reduce NET production in vitro $[8,9]$.

Breathomics provide one such opportunity for noninvasive diagnosis. Breath samples can be analysed on a variety of platforms. Gas chromatography mass spectrometry (GC-MS) is considered the gold standard and can distinguish individual VOCs. Field asymmetric ion mobility spectrometry (FAIMS), for example via Lonestar (Owlstone Medical, Cambridge, UK), relies on VOCs from a breath sample travelling through a charged chamber, and the pattern of migration according to ionic charge allows individual compounds to be distinguished. Electronic nose (eNose) devices are electronic systems containing chemoresistor sensors that generate an electric signal upon encountering a gaseous mixture. For example, the Cyranose (Sensigent, Baldwin Park, CA, USA) device has been used widely in the field, containing 32 composite polymer carbon black sensors that each generate a signal. By definition, eNose devices cannot distinguish individual VOCs, but may be useful for pattern recognition. While other platforms for breathomics exist, FAIMS and eNose methods are currently being explored widely in the clinical research setting. In the case of eNoses, these are portable and therefore can ultimately form point-of-care tests. FAIMS technology also has the potential to be miniaturised for point-of-care use if a pattern of VOCs of interest is established.

If breathomics are to be used for patient selection in clinical studies, they first need evaluation for feasibility in randomised controlled trials (RCTs). The advent of sample collection via sorbent tubes with the ReCIVA device (Owlstone Medical) has only recently enabled centralised VOC analyses [10] via storage and shipping of breath samples at room temperature, but sparse longitudinal data to inform trial design has limited adoption, and to date no RCTs have evaluated VOCs longitudinally. Furthermore, no study has looked at Haemophilus breath signals specifically. Several studies have found VOCs or eNose signals associated with COPD exacerbations [11, 12] with three eNose studies specifically elucidating breath signals associated with exacerbations caused by bacterial/viral infection [13-15]. However, no study has focused on Haemophilus or microbiome colonisation, and the eNose studies on viral/bacterial infections lacked the ability to determine specific VOCs.

We conducted pilot RCT primarily to study the effects of a CXCR2 antagonist, danirixin, the primary results for which have been published [16]. In this pilot RCT, our exploratory objectives were feasibility of measuring VOCs via multiple techniques, assessing relationships between VOCs and Haemophilus colonisation, and whether CXCR2 antagonism with danirixin altered lung microbiome composition in individuals with COPD.

\section{Methods}

In a double-blind RCT (NCT03250689) [16], participants were randomised (3:1) to receive danirixin $35 \mathrm{mg}$ twice daily or placebo for 14 days.

Participants were included if they were aged between 50 and 75 years, with a clinical diagnosis of COPD with mild to moderate airflow obstruction (post-bronchodilator forced expiratory volume in $1 \mathrm{~s}\left(\mathrm{FEV}_{1}\right) /$ forced vital capacity ratio $<0.7$ and $\mathrm{FEV}_{1} \geqslant 40 \%$ predicted at screening), had elevated sputum NETs based on screening assay for histone-elastase complexes of $>0.5$ units $\cdot \mathrm{mL}^{-1}$ sputum, and were current or former smokers with a minimum 10 pack-year history. Patients with lung diseases other than COPD or recent pneumonia were excluded from the study, and patients on medication known to impact NETs formation were also excluded, for example, use of phosphodiesterase-4 inhibitors [17]: roflumilast, crisaborole and apremilast, broad-spectrum phosphodiesterase inhibitors (e.g. theophylline), raloxifene and molecular-weight heparin. Additionally, systemic immunosuppressive medication, including current oral corticosteroids at a dose $>5 \mathrm{mg}$, concurrently or within 28 days preceding the screening visit, acute or chronic use of antibiotics, including macrolides for the prevention or treatment of COPD exacerbations were prohibited. Examples of chronic use include daily or two to three times per week for $\geqslant 3$ months. Prohibited medications related to danirixin specifically were oral or injectable CYP3A4 or BCRP substrates with narrow therapeutic index.

Patients meeting inclusion criteria were randomised and underwent key assessments, including spirometry, VOC sampling via a ReCIVA device, induced sputum (via up to 4\% nebulised saline) and venepuncture at screening, day 1, day 7 and day 14. Patients who failed screening still provided sputum and VOCs samples at the screening visit, though they were not randomised to dosing groups for further visits and did not provide any other sample type. Sputum and breath samples from screen failures were included in analyses. VOC samples were taken after $6 \mathrm{~h}$ of fasting on all visits; at the baseline visit subjects additionally provided an extra VOC sample $4 \mathrm{~h}$ after dosing. Participants who were current smokers were asked to refrain from smoking for at least $4 \mathrm{~h}$ prior to each visit. Sputum measurements included microbiome 
(profiled via 16S rRNA gene sequencing), NETs (immunoassays for histone elastase and DNA elastase complexes, confocal microscopy for sputum NET area), and sputum neutrophils using methods described previously [5]. Sputum samples were additionally assessed for quality via percentage of squamous cell and viable leukocyte counts, and a primary completer population defined on the basis of having "good" or "acceptable" quality sputum at baseline and day 14; the primary completer population was used for NETs analyses, although all sputum samples were used for the microbiome analyses. Four pairs of VOC samples were taken at each measurement time point, and were subject to measurement via three techniques at a centralised laboratory: GC-MS, FAIMS via Lonestar and an eNose device, Cyranose. The fourth VOC sample pair was analysed by GC-MS when possible (i.e. when the back-up was not needed due to failure of primary sample on any of the three techniques) to provide a replicate measurement to increase accuracy of VOC levels.

For the VOC samples, a quality control (QC) sample was run between every four patient samples, and background monitoring was carried out with a blank tube run after every four patient samples and after every QC sample. Blank tubes were clean tubes used to monitor potential carry-over from one sample to the next or incomplete desorption; neither was found to be an issue. VOC levels were corrected for analytical variation/instrument drift by normalisation to the average drift in intensity of a mixture of external standards, i.e. the QC samples. This method was found to be superior to normalisation methods using the breath samples themselves, such as scaling to total signal intensity, in reducing analytical variation. The QC samples were made by spiking on a fixed volume of a QC solution onto a clean sorbent tube and briefly purging with high-purity nitrogen. The QC solution was composed of a selection of chemicals meant to reflect classes of VOCs commonly found in breath all at fixed concentrations. Ambient background controls were not collected or used for background subtraction.

A thermal desorption GC-MS chromatogram was converted into a features list and automatic mapping was applied to identify a unique set of characteristics with subsequent visual inspection to check peak shape and retention time, and specificity of ions. All molecular features of interest (MFs) were run against the National Institute of Standards and Technology standard database; when match between library and compound was $>70 \%$, the MF was given a tentative ID.

The primary end-point for the study was change from baseline in sputum NETs as measured via histone elastase immunoassays, and although sample size was based on feasibility, we powered the study for a $70 \%$ probability of detecting a true reduction of $30 \%$ reduction in NETs. Changes in lung microbiome and VOCs were exploratory end-points.

To test relationships between Haemophilus and VOCs, principal component analysis, single-effects models and joint-effects models were done with the sklearn, statsmodels and cvglmnet packages, respectively, in Python v3.6 in June 2019. All analyses focused on samples taken at the screening visit and were cross-sectional across the entire patient population regardless of being screening pass/fail. The effect of screening pass/fail on subsequent findings was assessed by confounder analysis and was not found to have any significant effect. Outlier capping was performed independently on each molecular feature using Tukey fences prior to the single- and joint-effect analyses. In cases where patient provided two screening breath samples that were successfully analysed by GC-MS, single-effect regression models used a Huber sandwich variance estimator to allow for the inclusion of multiple samples per patient. Permutation testing was used to adjust MF p-values for multiple testing [18]. Joint-effects regression models were built on all MFs with unadjusted $\mathrm{p}<0.2$ from the single-effects analysis using least absolute shrinkage and selection operator (LASSO) regression. The shrinkage $(\lambda)$ parameter was estimated using leave-group-out cross-validation, each group being all samples from a single patient. Relative abundance of the Haemophilus genus was used as a continuous outcome variable. 16S rRNA gene PCR products were analysed using the QIIME pipeline (version 1.9.1) [19] and taxonomies were assigned using a closed reference alignment to the Greengenes 16S rRNA database (version 13_8). If identification was not possible at the genus level, the operational taxonomic unites (OTUs) were classified at a higher taxonomic level. OTUs with a maximum representation in a sample of $0.5 \%$ were excluded.

All participants provided written informed consent. The East of Scotland Research Ethics Service 1 (reference: 17/SS/0111) provided ethical approval for the study, which was carried out in accordance with the Declaration of Helsinki.

\section{Results}

Baseline characteristics

43 participants were screened and 19 randomised (14 danirixin, five placebo) out of a planned 32 (figure 1); the study was terminated early due to cessation of the danirixin development programme. Both treatment 


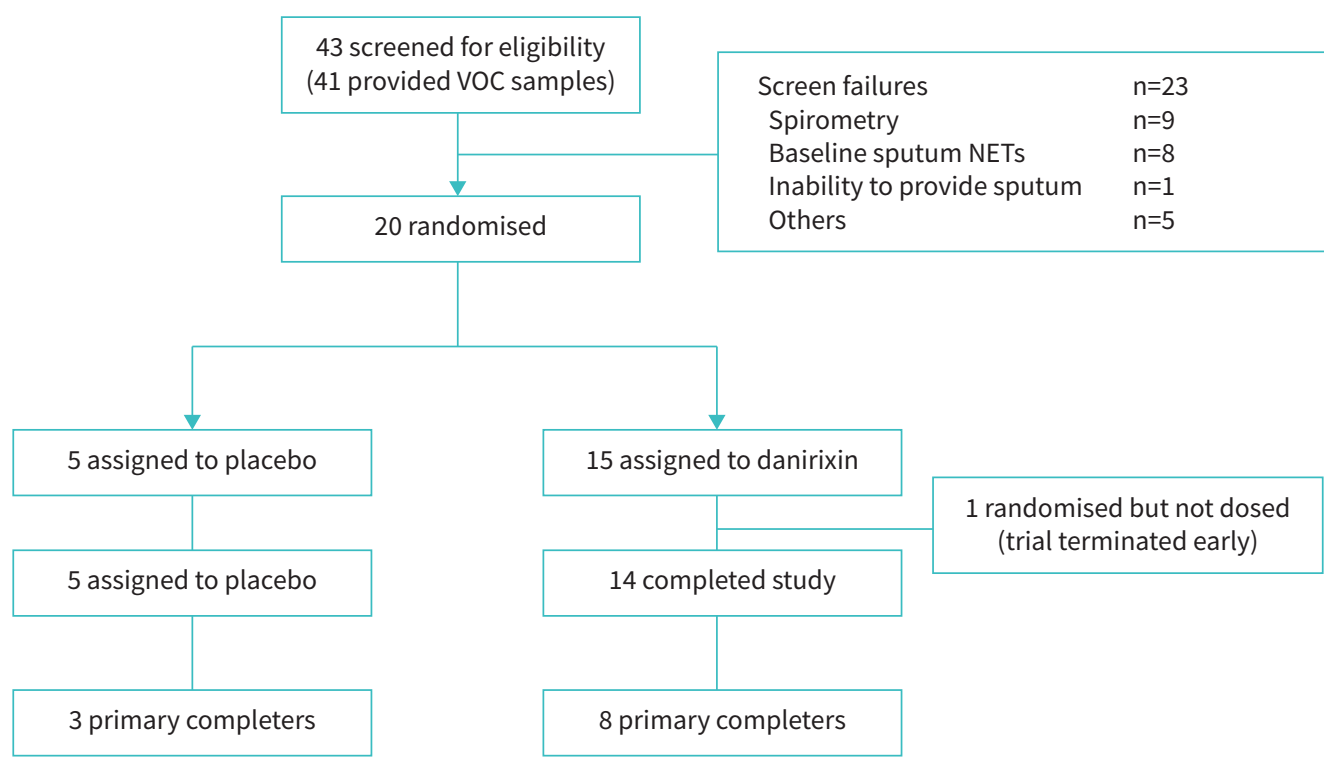

FIGURE 1 Consolidated Standards of Reporting Trials diagram for trial participants. The "primary completer" population was defined via subjects who provided "good"- or "acceptable"-quality sputum samples (based on percentage of squamous cells and viable leukocytes) at baseline and day 14. The primary completer population was used for sputum neutrophil extracellular traps (NETs) analyses, but the entire study population was used for the microbiome analysis. VOC: volatile organic compound.

groups were similar in terms of age and baseline $\mathrm{FEV}_{1}$ (table 1), although there was a greater proportion of current smokers in the placebo group (43\%) in comparison to the danirixin group (20\%).

VOC measures

For participants who failed screening, the VOC samples and sputum samples for the microbiome were included in the analyses for exploring the relationship between Haemophilus abundance and VOCs at the

\begin{tabular}{|c|c|c|}
\hline & Placebo & Danirixin \\
\hline Participants & 5 & 14 \\
\hline Age, years & $62 \pm 6$ & $65 \pm 7$ \\
\hline Male/female & $2 / 3$ & $6 / 8$ \\
\hline White/Caucasian/European & 5 & 14 \\
\hline Current smoker, \% & 2043 & 4320 \\
\hline Smoking history, pack-years & $48 \pm 13$ & $44 \pm 19$ \\
\hline Body mass index, $\mathrm{kg} \cdot \mathrm{m}^{-2}$ & 30.9 & 27.1 \\
\hline $\mathrm{FEV}_{1}, \mathrm{~L}$ & $2.49 \pm 0.64$ & $1.94 \pm 0.71$ \\
\hline $\mathrm{FEV}_{1}, \%$ predicted & $79.1 \pm 7.5$ & $69.5 \pm 18.4$ \\
\hline FVC, L & $4.07 \pm 1.16$ & $3.34 \pm 1.17$ \\
\hline $\mathrm{FEV}_{1} / \mathrm{FVC}$ & $0.62 \pm 0.08$ & $0.59 \pm 0.08$ \\
\hline CAT score & $17.0 \pm 1.00$ & $17.3 \pm 5.97$ \\
\hline \multicolumn{3}{|l|}{ Medications } \\
\hline Long-acting muscarinic antagonist & $3(60)$ & $9(64)$ \\
\hline Short-acting $\beta_{2}$-agonist & $4(80)$ & $8(57)$ \\
\hline Inhaled corticosteroid & $3(60)$ & $6(43)$ \\
\hline Long-acting $\beta_{2}$-agonist & $3(60)$ & $9(64)$ \\
\hline Systemic corticosteroid & 0 & $1(7)$ \\
\hline Anti-infectives & 0 & $1(7)$ \\
\hline
\end{tabular}


screening visit. There were 41 participants who provided VOC samples at the screening visit; only one participant was unable to provide sputum at screening.

Subsequent to screening, a primary completer population for the study was identified on the basis of acceptable sputum quality at both baseline and day 14. This resulted in three patients in the placebo arm and eight in the danirixin arm being part of the primary completer population for the purposes of measuring sputum NETs.

176 VOC samples were collected from 41 patients, but 68 samples were excluded at QC stage ( $\mathrm{n}=57$ due to detector saturation (thermal desorption), $n=7$ due to tube leak (thermal desorption), $n=1$ due to poor-quality in lab (machine maintenance), $\mathrm{n}=1$ due to low volume at collection) and six samples did not have matching microbiome analysis at screening. From the screening visit, this resulted in 31 samples from 22 patients. MFs or VOCs showed considerable variability in absolute levels longitudinally.

\section{Microbiome}

There were no statistically significant differences between treatment groups in microbiome $\alpha$-diversity, total bacterial load or relative Haemophilus abundance (figure 2). Lung microbiome composition appeared broadly similar to that seen in other COPD cohorts (figure 2), but abundance of Haemophilus was lower than that observed in COPD cohorts enriched for frequently exacerbating participants [20, 21].

\section{Relationship between individual VOCs and Haemophilus, sputum neutrophils and NETS}

Cross-sectional correlations between VOC levels and other factors were assessed using samples from the screening visit. GC-MS identified 105 MFs; a single-effects model for individual MFs identified four
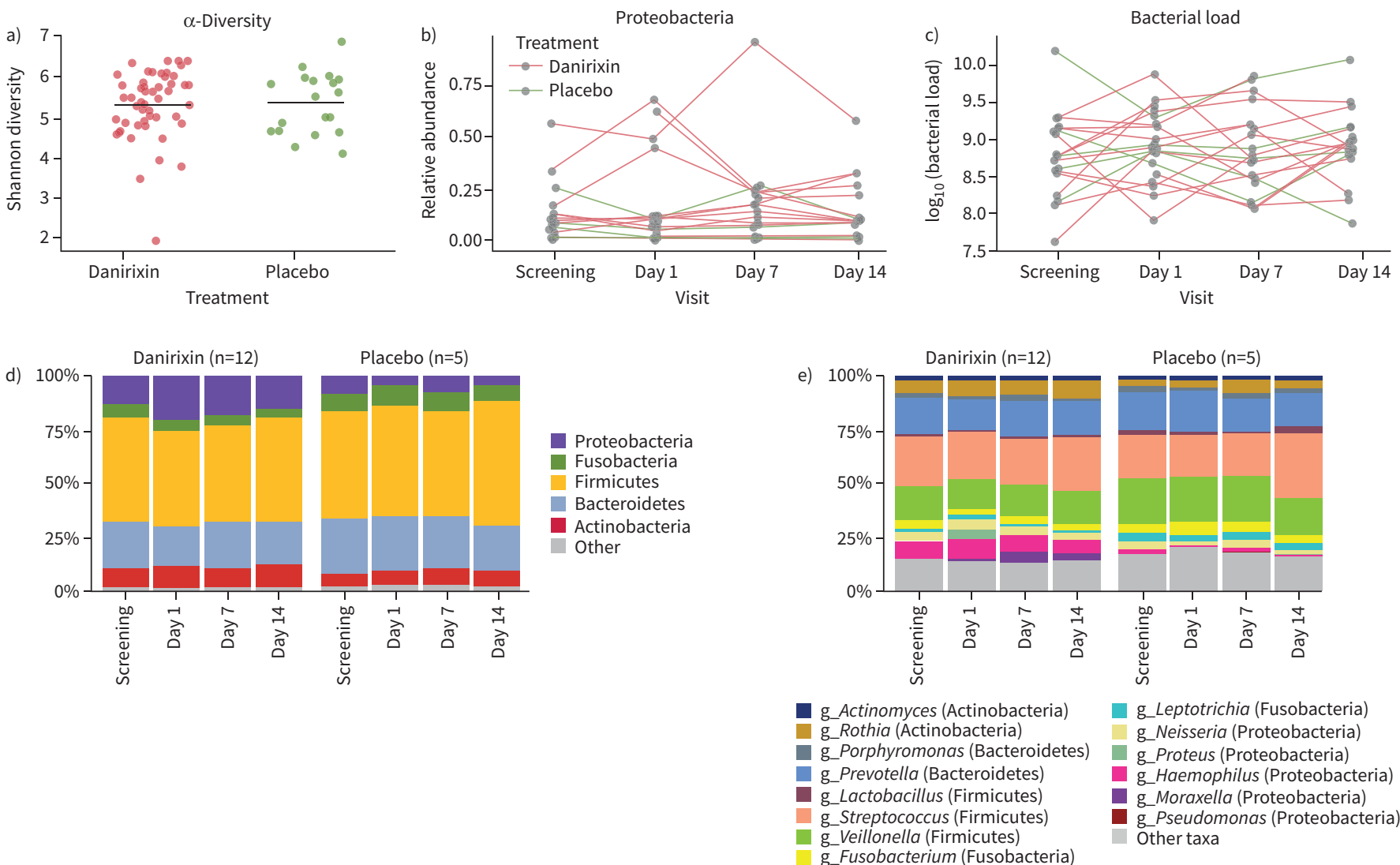

FIGURE 2 Changes in lung microbiome composition and bacterial load during study. a) (Shannon-) $\alpha$-diversity showed no significant differences by treatment group ( $p=0.858$, Wilcoxon rank-sum test) using pooled samples across visits between treatment groups. b) Changes in relative abundance of Proteobacteria (including Haemophilus) during the study. No significant differences in a linear mixed-effects (LME) model (using the patient as a random effect) were observed between danirixin $(n=12)$ and placebo $(n=5)$ groups $(p=0.174)$. $c)$ No significant differences in bacterial load as measured via $16 \mathrm{~S}$ quantitative PCR between danirixin and placebo groups were observed ( $p=0.8551, L M E)$. $d, e)$ Overall microbiome composition was similar between danirixin and placebo groups at the d) phylum and e) genus levels. 


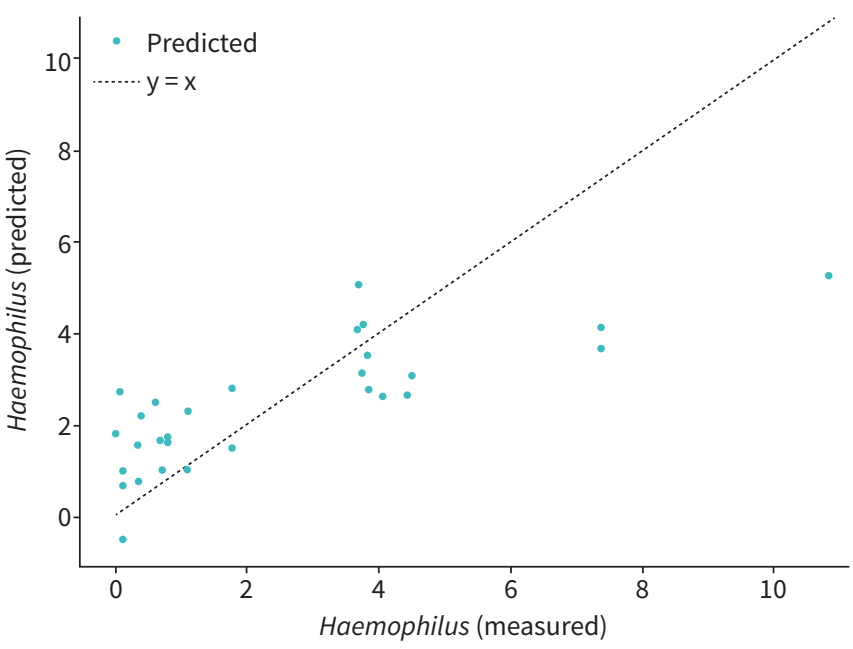

FIGURE 3 Joint-effects model to evaluate predictive ability of volatile organic compounds (VOCs) for Haemophilus influenzae relative abundance. Plot of predicted values for joint-effects model for VOCs against the measured values of Haemophilus relative abundance. Each point represents the predicted and measured value for a single sample at the screening visit $(n=31)$. The dashed line represents the line for a perfect model.

VOCs with significant correlations (R 0.15) with Haemophilus abundance. A joint-effects model with eight VOCs gave a modest correlation with Haemophilus $\left(\mathrm{R}^{2}=0.55\right)$ (figure 3). FAIMS identified 55 MFs; a single-effects model for Haemophilus identified one significant MF with poor correlation; and a joint-effects model could not be properly evaluated.

There was no overlap between VOCs that had the highest correlations with Haemophilus abundance, sputum neutrophils or NETs (figure 4); no significant correlation between individual VOCs and sputum neutrophils and NETs as measured via GC-MS or GC-FAIMS was observed, but the small number of paired samples available for these analyses limited definitive conclusions. VOCs or MFs that correlated most strongly with Haemophilus were different to those that correlated most strongly sputum NETs or neutrophils, suggesting distinct biological pathways and/or origins for these VOCs.

For Cyranose, 8.5\% of samples could not be analysed, as sensor data were abnormally low. Sensors displayed time trends unrelated to subject, treatment or visit; after July 2018 there was a noticeable decrease in mean and variance for all sensors, suggesting sensor drift. No relationship was observed between sensor signals and Haemophilus abundance across the population at baseline sputum neutrophils or NETs.

\section{Discussion}

Measuring exhaled VOCs is feasible in RCTs; however, backup samples should be taken along with stringent instrument monitoring due to the potential for QC failure. Individual VOCs may relate to Haemophilus colonisation, and a joint-effects model found a modest correlation between VOCs and Haemophilus relative abundance, but this relationship was only apparent via GC-MS analyses.

Three of the VOCs were tentatively identified as methylated hydrocarbons of similar chemical functionality to those previously associated with inflammatory conditions in human subjects, although different to hexane, nonanal and 1-propranolol recently identified as being related to eosinophilic asthma, and undecane, indicative of a pauci-granulocytic sputum phenotype [22]. Identified VOCs from our clinical in vivo samples were distinct from those reported in literature to be released in vitro by $H$. influenzae [23]; however, it is possible that some of the unidentified hydrocarbons may prove a match. In addition, the in vitro versus in vivo VOC profile may differ, since in vivo profiles will be modulated by other microbiota components in addition to other factors such as diet or airway inflammation. There is a paucity of data for disease-specific VOCs across the literature, with CHRISTIANSEN et al. [24] noting that no candidate breath biomarkers in COPD were detectable in all the studies in their literature review, and only three biomarkers were reported in more than one study. Thus, our present data add to the growing library of compounds that may be important in COPD and airways disease. 
a)

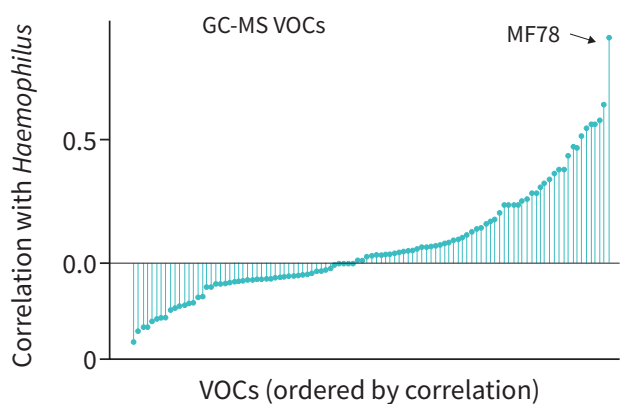

c)

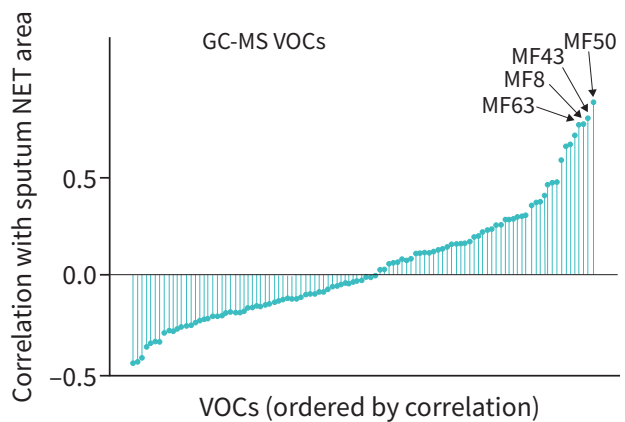

b)

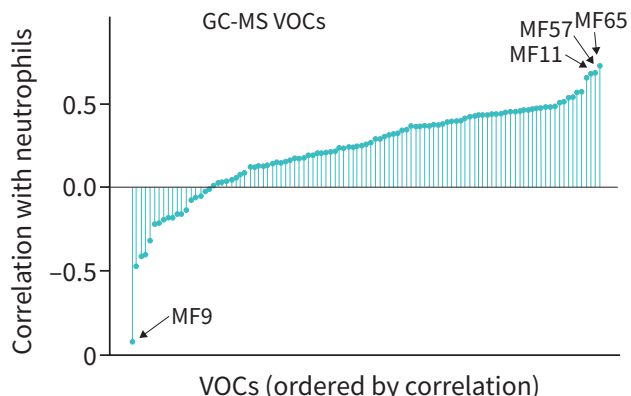

FIGURE 4 Individual volatile organic compounds (VOCs) ordered by correlation with Haemophilus influenzae relative abundance, percentage sputum neutrophils and sputum neutrophil extracellular traps (NETs) area as measured by gas chromatography mass spectrometry (GC-MS). Individual VOCs or molecular features of interest (MFs), measured by GC-MS, ordered by correlation against a) Haemophilus influenzae relative abundance; b) percentage sputum neutrophils; and c) sputum NET area. MF 78 has strongest correlation with Haemophilus influenzae relative abundance; MFs 65, 57 and 11 with percentage sputum neutrophils; and MFs 50, 43, 8 and 63 with sputum NET area, showing overall lack of overlap between VOCs that may relate to host microbiome, sputum neutrophils and sputum NETs.

One reason for the differing VOC profiles across literature may be the variability in absolute levels of compounds, which we observed in our own study, and has been noted even for established biomarkers. $F_{\text {eNO }}$ is an example of an exhaled compound that has successfully been implemented into clinical practice, and can used both clinically and in trials to identify patients with eosinophilic asthma. Despite becoming an established biomarker, $F_{\text {eNO }}$ still demonstrates considerable intra-day, intra-patient variability in terms of absolute levels [25]. Therefore, the longitudinal variability points to the need for standardised sampling protocols, since it appears that a period of fasting alone may not be enough. There is also an urgent need for identifying background VOCs for use as "housekeepers" to normalise levels of compounds against. Taking ambient background samples may also help to eliminate some sources of variation. In addition, our work with the Cyranose eNose device points to the need for considering calibration and drift, especially if considering use at the bedside for diagnosis.

Although sampling was acceptable to patients and site staff in our study, with overall high compliance with sampling, there was a notable rate of QC failure at the analysis stage. Since our study, further work has suggested that it may be acceptable to freeze breath samples, which may allow for backup samples to be taken and can mitigate failure at the analyses stage, although further validation work is required in this regard. Coupled with the high QC failure rate for the VOC samples and early trial termination, the limitation in paired sputum and VOC samples limited our ability to measure longitudinal relationships between Haemophilus abundance, sputum neutrophils or NETs and VOCs.

Study results were inconclusive in determining whether CXCR2 antagonism altered lung microbiome composition in COPD due to the early termination of the study; however, the 2-week treatment period was probably too short to expect changes in microbiome composition. Furthermore, we sampled induced sputum for microbiome, and subtle changes in the lower airway may be obscured by the high biomass 
from oral microbiome. The lower than anticipated sample size, high VOC sample failure rate and the lower than expected Haemophilus relative abundance limited the ability to detect a relationship between VOCs and Haemophilus at screening. While we included individuals with elevated sputum NETs, which may correlate with Haemophilus abundance [5], our trial participants had higher FEV $\mathrm{F}_{1}$ and were not enriched for frequent exacerbations [20, 21], which could explain the lower Haemophilus predominance in our study.

One limitation of our study was the imbalance between placebo and danirixin groups in baseline smoking status, which could lead to differences in both NET formation and VOCs. Participants were asked to refrain from smoking for $\geqslant 4 \mathrm{~h}$ prior to each visit, however that time limit may be of insufficient duration to impact VOCs. Furthermore, ongoing systemic inflammation from smoking between visits may impact NETs production. Only one subject in the danirixin group was on systemic steroids and anti-infectives during the study; therefore, this is unlikely to impact our overall results and conclusions. Additionally, we note that there is a lack of robust evidence that steroids impact NET production.

In conclusion, measuring exhaled VOCs is feasible in RCTs, and our results suggest that VOCs may relate to Haemophilus abundance. Several challenges remain for implementing breath analyses into RCTs, especially the longitudinal variability in individual VOC abundance. We recommend that GC-MS form part of any VOC evaluation, and that backup samples are taken in further exploration of the utility of VOCs as a diagnostic tool.

Acknowledgements: The authors would like to thank all study participants and members of the GSK and University of Dundee study teams during the conduct of the study.

Provenance: Submitted article, peer reviewed.

This study is registered at www.clinicaltrials.gov with identifier number NCT03250689

Data availability: Upon publication, anonymised individual participant data and study documents can be requested for further research from www.clinicalstudydatarequest.com

Conflict of interest: D. Mohan is a current employee and shareholder of Genentech/Roche. H.R. Keir has nothing to disclose. H. Richardson has nothing to disclose. D. Mayhew is a former employee of and shareholder in GSK. J. Boyer is a former employee of and shareholder in GSK. M.P. van der Schee is an employee of Owlstone Medical Ltd and holds options in the company. M.D. Allsworth is an employee of Owlstone Medical Ltd and holds options in the company. B.E. Miller is a former employee of and shareholder in GSK. R. Tal-Singer is a former employee of and shareholder in GSK, and has received personal fees from Immunomet, Vocalis Health and Ena Respiratory. J.D. Chalmers reports grants and personal fees from GSK during the conduct of the study; and research grants from Boehringer Ingelheim (BI), AstraZeneca (AZ), Gilead Sciences, Grifols and Insmed, and has received personal fees from BI, AZ, Chiesi, Grifols, Napp, Novartis, Insmed and Zambon.

Support statement: This study (GSK study 207551) was funded by GSK (NCT03250689). Funding information for this article has been deposited with the Crossref Funder Registry.

\section{References}

1 Menzies-Gow A, Mansur AH, Brightling CE. Clinical utility of fractional exhaled nitric oxide in severe asthma management. Eur Respir J 2020; 55: 1901633.

2 Gaida A, Holz O, Nell C, et al. A dual center study to compare breath volatile organic compounds from smokers and non-smokers with and without COPD. J Breath Res 2016; 10: 026006.

3 Phillips CO, Syed Y, Parthaláin NM, et al. Machine learning methods on exhaled volatile organic compounds for distinguishing COPD patients from healthy controls. J Breath Res 2012; 6: 036003.

4 Finamore $\mathrm{P}$, Pedone $\mathrm{C}$, Scarlata S, et al. Validation of exhaled volatile organic compounds analysis using electronic nose as index of COPD severity. Int J Chron Obstruct Pulmon Dis 2018; 13: 1441-1448.

5 Dicker AJ, Crichton ML, Pumphrey EG, et al. Neutrophil extracellular traps are associated with disease severity and microbiota diversity in patients with chronic obstructive pulmonary disease. J Allergy Clin Immunol 2018; 141: 117-127.

6 Brinkmann V, Reichard U, Goosmann C, et al. Neutrophil extracellular traps kill bacteria. Science 2004; 303: 1532-1535.

7 Narasaraju T, Yang E, Samy RP, et al. Excessive neutrophils and neutrophil extracellular traps contribute to acute lung injury of influenza pneumonitis. Am J Pathol 2011; 179: 199-210. 
8 Lazaar AL, Miller BE, Tabberer M, et al. Effect of the CXCR2 antagonist danirixin on symptoms and health status in COPD. Eur Respir J 2018; 52: 1801020.

9 Pedersen F, Waschki B, Marwitz S, et al. Neutrophil extracellular trap formation is regulated by CXCR2 in COPD neutrophils. Eur Respir J 2018; 51: 1700970.

10 van der Schee MP, Fens N, Brinkman P, et al. Effect of transportation and storage using sorbent tubes of exhaled breath samples on diagnostic accuracy of electronic nose analysis. J Breath Res 2013; 7: 016002.

11 Gaugg MT, Nussbaumer-Ochsner Y, Bregy L, et al. Real-time breath analysis reveals specific metabolic signatures of COPD exacerbations. Chest 2019; 156: 269-276.

12 Pizzini A, Filipiak W, Wille J, et al. Analysis of volatile organic compounds in the breath of patients with stable or acute exacerbation of chronic obstructive pulmonary disease. J Breath Res 2018; 12: 036002.

13 Lewis JM, Savage RS, Beeching NJ, et al. Identifying volatile metabolite signatures for the diagnosis of bacterial respiratory tract infection using electronic nose technology: a pilot study. PLoS One 2017; 12: e0188879.

14 van Geffen WH, Bruins M, Kerstjens HA. Diagnosing viral and bacterial respiratory infections in acute COPD exacerbations by an electronic nose: a pilot study. J Breath Res 2016; 10: 036001.

15 Shafiek H, Fiorentino F, Merino JL, et al. Using the electronic nose to identify airway infection during COPD exacerbations. PLoS One 2015; 10: e0135199.

16 Keir HR, Richardson H, Fillmore C, et al. CXCL-8-dependent and -independent neutrophil activation in COPD: experiences from a pilot study of the CXCR2 antagonist danirixin. ERJ Open Res 2020; 6: 00583-2020.

17 Totani L, Amore C, Di Santo A, et al. Roflumilast inhibits leukocyte-platelet interactions and prevents the prothrombotic functions of polymorphonuclear leukocytes and monocytes. J Thromb Haemost 2016; 14 191-204.

18 Churchill GA, Doerge RW. Empirical threshold values for quantitative trait mapping. Genetics 1994; 138 : 963-971.

19 Caporaso JG, Kuczynski J, Stombaugh J, et al. QIIME allows analysis of high-throughput community sequencing data. Nat Methods 2010; 7: 335-336.

20 Mayhew D, Devos N, Lambert C, et al. Longitudinal profiling of the lung microbiome in the AERIS study demonstrates repeatability of bacterial and eosinophilic COPD exacerbations. Thorax 2018; 73: 422-430.

21 Wang Z, Bafadhel M, Haldar K, et al. Lung microbiome dynamics in COPD exacerbations. Eur Respir J 2016; 47: 1082-1092.

22 Schleich FN, Zanella D, Stefanuto PH, et al. Exhaled volatile organic compounds are able to discriminate between neutrophilic and eosinophilic asthma. Am J Respir Crit Care Med 2019; 200: 444-453.

23 Filipiak W, Sponring A, Baur MM, et al. Characterization of volatile metabolites taken up by or released from Streptococcus pneumoniae and Haemophilus influenzae by using GC-MS. Microbiology 2012; 158: 3044-3053.

24 Christiansen A, Davidsen JR, Titlestad I, et al. A systematic review of breath analysis and detection of volatile organic compounds in COPD. J Breath Res 2016; 10: 034002.

25 Wang R, Alahmadi F, Niven R, et al. Same-day repeatability of fractional exhaled nitric oxide in severe asthma. Eur Respir J 2021; 57: 2003391. 